\title{
Improved Parallel PSO Inversion for the MT Sounding Data
}

\author{
Jie Xiong \\ School of Electronics and Information \\ Yangtze University \\ Jingzhou, China
}

\author{
Caiyun Liu \\ School of Mathematics and Information \\ Yangtze University \\ Jingzhou, China
}

\begin{abstract}
The magnetotelluric (MT) method has become more widely used in hydrocarbon exploration. The inversion of MT data, which can determine the electrical structure of subsurface, is a nonlinear and multimodal optimization problem. Particle swarm optimization (PSO) algorithm is a good solver for this geophysical inversion problem, whereas it has a shortage of heavy computation time. An improved parallel adaptive PSO inversion algorithm for MT data is proposed in order to decrease the computation time. The performance of the proposed algorithm was evaluated on the Dawn 4000L supercomputer using the synthetic MT data of 1D layered geo-electrical models of three and four layers. The numeric results show that the proposed algorithm can obtain the as good solution as the serious PSO inversion algorithm, and can reduce the computation time obviously when more computing nodes been employed. This result indicates that proposed improved parallel inversion algorithm can deal with the computation time problem and provide theory and technology support the MT data non-linear inversion based on PSO.
\end{abstract}

Keywords- improved parallel PSO; non-linear inversion; Magnetotelluric (MT); coarse granularity

\section{INTRODUCTION}

The magnetotelluric (MT) method utilizes naturally occurring low-frequency electro-magnetic waves to determine the electrical resistivity of the Earth's subsurface. A knowledge of the resistivity is important since rock types important to hydrocarbon exploration can be differentiated on the basis of resistivity value $[1,2]$. It has some advantages such as low cost, large probing depth, not affected by high-resistivity shielding and high resolution to the low-resistivity layer. In the last decades, the MT method has become more widely used in hydrocarbon exploration [3].

Data inversion is one of the core issues of the MT method, which is classified into linear and nonlinear categories. To overcome the flaws of the classical linear inversion method, some nonlinear inversion methods, such as Monte Carlo, simulated annealing and genetic algorithm are employed to the MT inversion [4]. However, they have some disadvantages [2].

The particle swarm optimization (PSO) is a relative novel global optimization algorithm based on swarm intelligence [5]. It has several advantages including fast convergence, strong capability of global optimization and simple parameter adjustment, and has been successfully applied to many fields [6]. In recent years it has also been introduced into the geophysical inversion [7-11]. Although the disadvantage, such as local optimum, of geophysical inversion based on PSO has been improved by employing the adaptive inertial weight, it still has the problem of heavy computation time when the forward modeling becomes more complex [2]. Parallel implementation can reduce the computation time of inversion obviously.

Message Passing Interface (MPI) is one of the most popular parallel computing software environment [12]. An asynchronous parallel adaptive PSO inversion algorithm based on MPI for the MT oil-gas exploration data is proposed in this paper. It is applied to the inversion of the synthetic MT data and its parallel performance is analyzed.

\section{MethodOLOGY}

\section{A. Forward modeling}

Assume an 1D layered model through a cross section of $n$ layers, of which from up to down the resistivities are $\rho_{1}, \rho_{2}, \ldots, \rho_{n}$, respectively, and the depths are $h_{1}, h_{2}, \ldots, h_{n}$, respectively, where $h_{n}=\infty$. For such a 1D layered model, apparent resistivity $\rho_{a}$ and phase $\phi_{a}$ can be calculated as follows:

$$
\rho_{a}(\omega)=\frac{|Z(\omega)|^{2}}{\omega \mu}, \quad \phi_{a}=\arctan \frac{\operatorname{Im}(Z)}{\operatorname{Re}(Z)}
$$

Where $\omega=2 \pi T$ is angular frequency, $\mu$ is magnetic permeability, $Z(\omega)$ is wave impedance on the surface that can be calculated by the following recurrence formula:

$$
Z_{i}=Z_{0 i} \frac{Z_{0 i}\left(1-e^{-2 k_{i} h_{i}}\right)+Z_{i+1}\left(1+e^{-2 k_{i} h_{i}}\right)}{Z_{0 i}\left(1+e^{-2 k_{i} h_{i}}\right)+Z_{i+1}\left(1-e^{-2 k_{i} h_{i}}\right)}, Z_{N}=\frac{\omega \mu}{k_{N}}=Z_{0 N}
$$

Where $k_{i}=\sqrt{i \omega \mu / \rho_{i}}$ is the complex wave numbers of the $i$-th layer, $Z_{0 i}$ is the characteristic impedance of the $i$-th layer, and $Z_{i}$ is the wave impedance of the top of the $i$-th layer. 
The presentation above indicates that apparent resistivity and phase on N-layer geo-electrical section can be expressed by functions of signal periods and section parameters:

$$
\begin{aligned}
& \rho_{a}=f_{1}\left(\rho_{1}, \rho_{2}, \ldots, \rho_{N}, h_{1}, h_{2}, \ldots, h_{N-1}, T\right) \\
& \phi_{a}=f_{2}\left(\rho_{1}, \rho_{2}, \ldots, \rho_{N}, h_{1}, h_{2}, \ldots, h_{N-1}, T\right)
\end{aligned}
$$

\section{B. Improved PSO}

In the PSO algorithm [5], the optimal solution is obtained through collaboration among individual which represented by particles. Suppose that the search space is of $\mathrm{n}$ dimensions, total number of particles is $\mathrm{m}$, the position of the $i$-th particle in the $n$-dimensional space is xi, and its flight speed is vi. Each particle has an adaptive value determined by an optimization target function, and is aware of the best position pi that is found till now by itself and its current position, as well as the optimal position pg of the whole swarm found till present. Then the speed and the position of each particle can be updated by the following formulas:

$$
v_{i}^{k+1}=w v_{i}^{k}+c_{1} r_{1}\left(p_{i}-x_{i}^{k}\right)+c_{2} r_{2}\left(p_{g}-x_{i}^{k}\right)
$$

$$
x_{i}^{k+1}=x_{i}^{k}+v_{i}^{k+1}
$$

where $v_{i}^{k}$ is the flight velocity of the $i$-th particle after $k$-th iteration, $x_{i}^{k}$ is the position of the $i$-th particle after $k$-th iteration, $r_{1}$ and $r_{2}$ are random parameters evenly distributed in $0 \sim 1, c_{1}$ and $c_{2}$ are weight factors, and $w$ is inertial weight which is important to the balance between global exploration and local exploitation.

The Adaptive PSO [2] adjust the inertial weight dynamically according to the particle velocity of the population as follows:

$$
\begin{aligned}
& \text { if } v_{\text {avg }}^{k}>v_{e}^{k} \text {, then } w(k+1)=w(k) / p ; \\
& \text { if } \quad v_{a v g}^{k}<v_{e}^{k}, \quad \text { then } \quad w(k+1)=w(k) * p ;
\end{aligned}
$$

$$
\text { if } v_{\text {avg }}^{k}=v_{e}^{k} \text {, then } w(k+1)=w(k),
$$

where

$v_{\text {avg }}^{k}=\left(\sum_{i=1}^{m} \sum_{j=1}^{n}\left|v_{i j}^{k}\right|\right) /\left(m^{*} n\right)$

is the average velocity of the $k$-th generation population,

$v_{e}^{k}=v_{0} e^{-\left(2 k /\left(T_{\max }-T_{1}\right)\right)^{2}}$

is the expected velocity of the $k$-th generation population which attenuates exponentially, here $T_{\max }$ is the maximum evolution iteration, $T_{1}$ is the local exploitation iteration,
$T_{\max }-T_{1}$ the iteration of global exploration, and parameter $p$ is the change rate of inertial weight.

\section{Parallel Strategy}

Some results [12] reported show that the coarse granularity is more effective then the fine granularity, because it can reduce the communication time so as to gain better performance. So we choose the coarse granularity in our parallel implementation of inversion, that is to say we assign a number of particles to one processor.

We regard the particles in the same processor as an independent sub-swarm, which has the full information. The particles in the same processor (sub-swarm) can construct the optimal solution independently guided by their own information. Different sub-swarm (different nodes) interact each other by exchanging the information such as sub-swarm optimal solution. We name the dividing strategy as coarse granularity interacting multi particle swarms.

In order to reduce the communication time of parallel computing, we choose the asynchronous parallel strategy, which means each node (sub-swarm) run certain iterations locally and exchange the information each other controlled by the master node and then begin the next certain iterations.

\section{Improved Parallel PSO inversion algorithm}

The forward model can be written as $d=A(m)$, where $m$ is a model parameter, A is forward functional, and $d$ is the theoretical value corresponding to the model $\mathrm{m}$. Inversion is to solve the model parameter $m$ from the observed value $d^{o b s}$, which makes the fitness error between the theoretical value $d=A(m)$ and the observed value $d^{\text {obs }}$ least. The objective function of inversion is defined as the norm $L_{2}$ of the difference between the observed and theoretical values to describe the fitness degree, which is expressed as

$$
P(m)=\left\|d^{o b s}-A(m)\right\|^{2} \rightarrow \min
$$

For an N-layer geo-electrical section, the model parameter $\mathrm{m}$ is a (2N-1) dimensional vector $\left(\rho_{1}, \rho_{2}, \ldots, \rho_{N}, h_{1}, h_{2}, \ldots, h_{N-1}\right)^{T}$. If the observation is conducted at $K$ frequencies, the observed value $d^{\text {obs }}$ will is a $2 * K$ dimensional vector $\left(\rho_{1}, \rho_{2}, \ldots \rho_{K}, \phi_{1}, \phi_{2}, \ldots \phi_{k}\right)^{T}$, where $\rho_{i}, \phi_{i}$ are apparent resistivity and impedance phase at the i-th frequency. The inversion of MT data for 1D N-layer model is to find the model parameter $m=\left(\rho_{1}, \rho_{2}, \ldots, \rho_{N}, h_{1}, h_{2}, \ldots, h_{N-1}\right)^{T}$, which results a best match between the calculated value $d=A(m)$ from Eqs. (3) and (4) and the observed value $d^{o b s}$.

The improved parallel PSO inversion algorithm is described as follow: 
Step 1: Input node number (sub-swarm number) N, particle number $\mathrm{S}$ of each sub-swarm;

Step 2: Master node generate $N * S$ parameters of initial model randomly, corresponding to initial positions of $\mathrm{N}^{*} \mathrm{~S}$ particles, and send $i$-th sub-swarm to $i$-th node;

Step 3: Each node calculates each particle's theoretical value $A(m)$ of their own sub-swarm respectively, using formulas (3) and (4);

Step 4: Each node calculates objective function value of each particle of their own sub-swarm by formula (8);

Step 5: Each node selects the best particle of their own subswarm;

Step 6: Each node calculate current average velocity $v_{a v g}$ of sub-swarm, and adjust inertial weight $w$ of sub-swarm dynamically according to $v_{\text {avg }}$ and $\mathrm{v}_{\mathrm{e}}$ by formula (7);

Step 7: Each node adjusts the position and velocity of each particle of their own sub-swarm, according to following formulas (5) and (6);

Step 8: If every nodes runs certain iterations, they exchange the information such as the position and velocity of the best particle of each sub-swarm, controlled by the master node;

Step 9: if global convergence or maximum number of iteration is met, go to Step 10, otherwise return to Step 3 to perform next iteration;

Step 10: Master node output result of inversion, finish calculation.

\section{NUMERICAL RESULT AND ANALYSIS}

The parallel PSO inversion algorithm is conducted to the noise-free MT data, with 20 particles, maximum iteration number 2000 and learning factor $\mathrm{c} 1=\mathrm{c} 2=2$. In inversion, the value ranges taken are $\rho_{1}=0 \sim 500 \Omega \mathrm{m}, \rho_{2}=0 \sim 100 \Omega \mathrm{m}, \rho_{3}=$ 0 4000 $\Omega \mathrm{m}, h_{1}=0 \sim 2000 \mathrm{~m}, h_{2}=0 \sim 4000 \mathrm{~m}$, respectively. The inversion result is shown in Table 1, where the sum of relative errors is that of inversion model parameters $\left(\rho_{1}, \rho_{2}, \rho_{3}, h_{1}, h_{2}\right)$ and real model parameters.

MT data inversion by parallel PSO is made on the fourlayer (type $\mathrm{HH}$ ) model. The result is listed in Table 2. In inversion, 20 particles, maximum iteration number 2000 and learning factor $\mathrm{c} 1=\mathrm{c} 2=2$ are adopted, and the value ranges taken are $\rho_{1}=0 \sim 1000 \Omega \mathrm{m}, \rho_{2}=0 \sim 1000 \Omega \mathrm{m}, \rho_{3}=0 \sim 1000 \Omega \mathrm{m}$,

$\rho_{4}=0 \sim 1000 \Omega \mathrm{m}, h_{1}=0 \sim 4000 \mathrm{~m}, h_{2}=0 \sim 4000 \mathrm{~m}, h_{3}=0 \sim 4000 \mathrm{~m}$, respectively. In these ranges, 20 initial particles are generated randomly for iterative inversion.

Tables 1 and 2 show the accuracy of result of parallel inversion is as well as that of serial one's but it spends much less computation time.

We plot the speedup and speedup efficiency of parallel PSO inversion algorithm in figure 2 following the data of Table 2. From Fig. 2, we can find that more computation nodes lead to less computation time but less speedup efficiency, for inversion.

TABLE I. PARALLEL INVERSION RESULTS ON THE THREE-LAYER MODEL

\begin{tabular}{|c|c|c|c|c|c|}
\hline Model & \multicolumn{4}{l|}{$\begin{array}{l}\text { Real } \\
\text { Parameter }\end{array}$} & \multicolumn{4}{l|}{ MT Inversion } \\
\cline { 3 - 6 } & 1 & $\begin{array}{l}1 \\
\text { node }\end{array}$ & $\begin{array}{l}10 \\
\text { nodes }\end{array}$ & $\begin{array}{l}\text { 20 } \\
\text { nodes }\end{array}$ & $\begin{array}{l}30 \\
\text { nodes }\end{array}$ \\
\hline$\rho 1$ & 110 & 110.1 & 110.0 & 109.9 & 109.7 \\
\hline$\rho 2$ & 20 & 20.01 & 20.0 & 20.43 & 20.1 \\
\hline$\rho 3$ & 1200 & 1199.8 & 1200.0 & 1200.2 & 1199.8 \\
\hline h1 & 500 & 498.6 & 498.9 & 498.2 & 500.2 \\
\hline h2 & 2000 & 2001.3 & 2001.6 & 2015.7 & 2017.3 \\
\hline $\begin{array}{c}\text { Running } \\
\text { number }\end{array}$ & -- & 50 & 50 & 50 & 50 \\
\hline $\begin{array}{c}\text { Calculation } \\
\text { time(s) }\end{array}$ & -- & 482.9 & 129.4 & 90.9 & 75.3 \\
\hline
\end{tabular}

TABLE II. PARALLEL INVERSION RESULTS ON THE FOUR-LAYER MODEL

\begin{tabular}{|c|c|c|c|c|c|}
\hline \multirow{2}{*}{$\begin{array}{l}\text { Model } \\
\text { Parameter }\end{array}$} & \multirow{2}{*}{$\begin{array}{l}\text { Real } \\
\text { Mode } \\
1\end{array}$} & \multicolumn{4}{|c|}{ MT Inversion } \\
\hline & & $\begin{array}{l}1 \\
\text { node }\end{array}$ & $\begin{array}{l}10 \\
\text { nodes }\end{array}$ & $\begin{array}{l}20 \\
\text { nodes }\end{array}$ & $\begin{array}{l}30 \\
\text { nodes }\end{array}$ \\
\hline$\rho 1$ & 100 & 99.9 & 100.2 & 99.9 & 99.9 \\
\hline$\rho 2$ & 20 & 19.2 & 20.6 & 20.0 & 19.9 \\
\hline$\rho 3$ & 300 & 281.6 & 300.4 & 286.5 & 319.4 \\
\hline $\mathrm{P} 4$ & 10 & 10.0 & 10.4 & 9.9 & 10.1 \\
\hline h1 & 600 & 612.1 & 595.9 & 599.5 & 601.4 \\
\hline $\mathrm{h} 2$ & 1500 & 1468.4 & 1502.6 & 1537.2 & 1512.1 \\
\hline h3 & 3000 & 3176.9 & 2899.2 & 3018.3 & 2979.4 \\
\hline $\begin{array}{l}\text { Running } \\
\text { number }\end{array}$ & -- & 50 & 50 & 50 & 50 \\
\hline $\begin{array}{c}\text { Calculation } \\
\text { time(s) }\end{array}$ & -- & 617 & 132.2 & 93.8 & 79.8 \\
\hline
\end{tabular}

\section{CONCLUSION}

The MT method has become more widely used in hydrocarbon exploration. In order to avoid the disadvantage of heavy computation cost, a synchronous parallel adaptive PSO inversion algorithm is proposed based on MPI. The performance of the proposed algorithm was evaluated on the Dawn 4000L supercomputer using the synthetic MT data of 1D layered geo-electrical models of three and four layers.

The numeric experiments shows that the proposed parallel inversion algorithm can obtain the as good inversion solution as serious version, and can reduce the computation time obviously when more computing nodes been used. This result indicates that proposed asynchronous parallel inversion algorithm can deal with the computation time problem and 
provide theory and technology support the MT data non-linear inversion based on PSO.
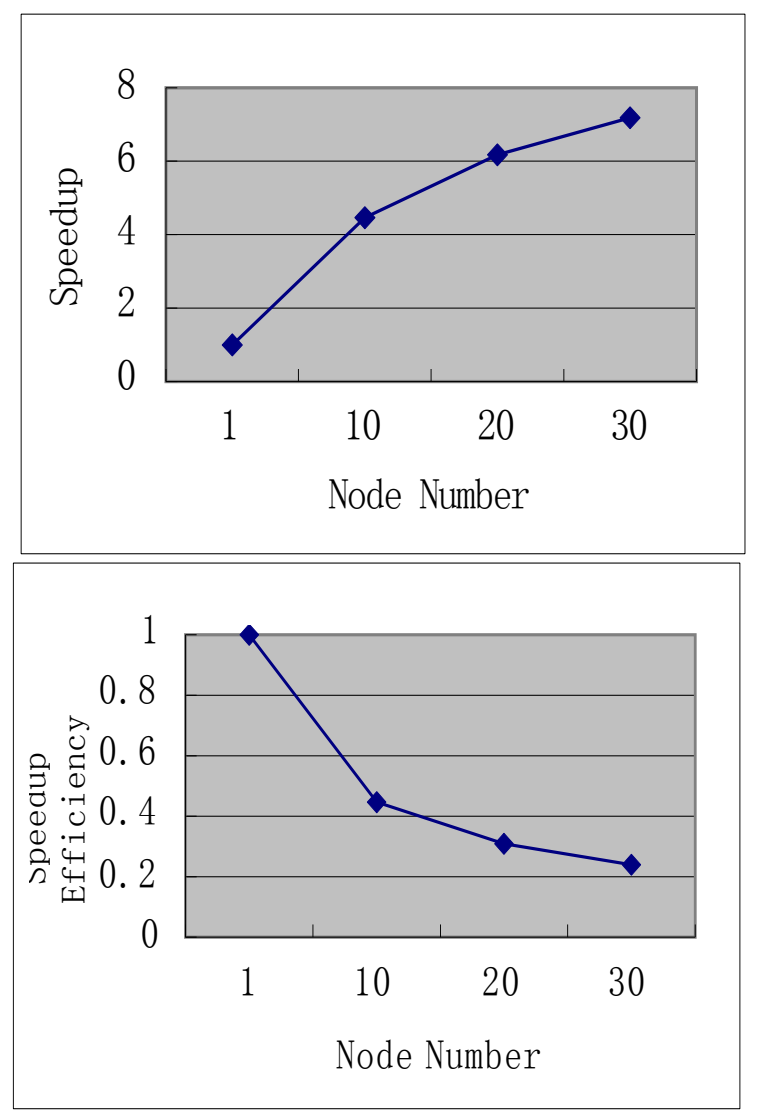

Figure 1. THE SPEEDUP AND SPEEDUP EFFICIENCY OF FOUR LAYER MT DATA PARALLEL INVERSION.

\section{ACKNOWLEDGMENT}

This work is support by the National Science Foundation of China (No. 61273179, No. 61673006), and Science and Technology Research Project of Education Department of Hubei Province of China (No. D20131206, No. B2016034, No. 20141304).

\section{REFERENCES}

[1] M. Cuma, A. Gribenko, and M. S. Zhdanov, "Inversion of magnetotelluric data using integral equation approach with variable sensitivity domain: Application to EarthScope MT data”, Physics of the Earth and Planetary Interiors, Vol.270, Sept. 2017, pp.113-127.

[2] X. Ogaya, J. Ledo, P. Queralt, et al., "A layer stripping approach for monitoring resistivity variations using surface magnetotelluric responses", Journal of Applied Geophysics, Vol. 132, Sept. 2016, pp.100-115.

[3] M. M. Sarvandani, A. N. Kalateh, M. Unsworth, et al., "Interpretation of magnetotelluric data from the Gachsaran oil field using sharp boundary inversion", Journal of Petroleum Science and Engineering, Vol. 149, Jan. 2017, pp. 25-39.

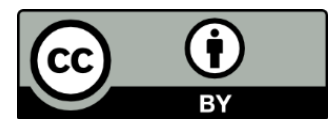

(C) 2017 by the author(s); licensee Empirical Research Press Ltd. United Kingdom. This is an open access article distributed under the terms and conditions of the Creative Commons by Attribution (CC-BY) license. (http://creativecommons.org/licenses/by/4.0/).
[4] J. Xiong, C. Liu, T. Zhang, et al., "A non-linear inversion algorithm based on adaptive PSO for the MT oil-gas exploration data", Energy Education Science and Technology Part A. Energy Science and Research", Vol. 32, No. 1, 2014, pp.473-480.

[5] J. Kennedy, R.C. Eberhart, "Particle Swarm Optimization", IEEE International Conference on Neural Netwowks. NJ: IEEE Service Center, 1995.

[6] M. R. Bonyadi, Z. Michalewicz, "Particle Swarm Optimization for Single Objective Continuous Space Problems: A Review", Evolutionary Computation, Vol.25, No. 1, 2016, pp. 1-54.

[7] J. L. Fernandez-Martinez, E. G. Gonzalo, J. P. F. Alvarez, et al., "PSO: A powerful algorithm to solve geophysical inverse problems: Application to a 1D-DC resistivity case", Journal of Applied Geophysics, Vol. 71, No. 1, 2010, pp. 13-25.

[8] S. Yuan, S. Wang, T. Nan, "Swarm intelligence optimization and its application in geophysical data inversion." Applied Geophysics Vol. 6, No. 2, 2009, pp.166-174.

[9] R. Shaw, S. Srivastava, "particle swarm optimization: A new tool to invert geophysical data.", Geophysics, Vol 72, No. 2, 2007, pp.F75-83.

[10] X. M. Shi, M. Xiao, J. K. Fan, et al., "The damped PSO algorithm and its application for magnetotelluric sounding data inversion.", Vol, 52, No, 4, 2009, pp.1114-1120.

[11] J.L.G. Pallero, J.L. Fernandez-Martinez, S. Bonvalot, et al., "3D gravity inversion and uncertainty assessment of basement relief via Particle Swarm Optimization", Journal of Applied Geophysics, Vol. 139, April, 2017, pp. 338-350.

[12] J. Xiong, C. Liu, Z. Chen, A New Parallel Ant Colony Optimization Algorithm Based on Message Passing Interface.”, IEEE PACIIA, 2008, p.178-182.

\section{AUTHORS PROFILE}

Jie Xiong received his Ph.D. Degree in Geophysics and Information Technology from China University of Geosciences in 2012. He currently is an Associate Professor at School of Electronics and Information, Yangtze University, China. His research interests include geophysical inversion theory, cloud computing, and scientific visualization.

Caiyun Liu (corresponding author) received her Ph.D. Degree in Geophysics and Information Technology from China University of Geosciences in 2014. She currently is an Associate Professor at School of Mathematics and Information, Yangtze University, China. Her research interests include applied geophysics, wavelet analysis, and optimization theory.
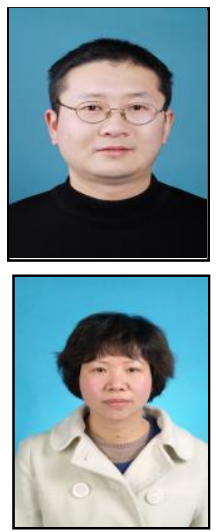